\title{
On Extremal Graphs With No Long Paths
}

\author{
Asad Ali Ali \\ Department of Computer Science \\ University of Mississippi \\ University, MS 38677 \\ ali@sam.cs.olemiss.edu
}

\author{
William Staton \\ Department of Mathematics \\ University of Mississippi \\ University, MS 38677 \\ mmstaton@cypress.mcsr.olemiss.edu
}

Submitted: October 31, 1995; Accepted: June 24, 1996

\begin{abstract}
Connected graphs with minimum degree $\delta$ and at least $2 \delta+1$ vertices have paths with at least $2 \delta+1$ vertices. We provide a characterization of all such graphs which have no longer paths.
\end{abstract}

Extremal problems involving paths and cycles have been considered since the infancy of graph theory. The question which interests us here is the question of what minimum degree condition guarantees a path of a preassigned length. This question was answered by Erdös and Gallai [4] and again by Andrasfai [1]. Our formulation of their answer is

Theorem 1 Let $G$ be a connected graph with minimum degree $\delta$ and at least $2 \delta+1$ vertices. Then $G$ contains a path of at least $2 \delta+1$ vertices.

The complete bipartite graphs $K_{\delta, n-\delta}$ with $n \geq 2 \delta+1$ show that the theorem is best possible in the sense that there exist graphs of minimum degree $\delta$ with no longer paths. Our purpose in this article is to characterize all such extremal graphs.

For a graph $\mathrm{G}$ with n vertices we define $f(G)$ to be the number of vertices in a longest path of G, and for a vertex $v$ of $\mathrm{G}$ we define $f(v)$ to be the number of vertices in a longest path of $\mathrm{G}$ with initial vertex $v$. A set of vertices is called independent if no two of them are adjacent. By the sum $H \oplus K$ of two graphs we mean the graph obtained from the disjoint union of $\mathrm{H}$ and $\mathrm{K}$ by adding edges joining every vertex of 
$\mathrm{H}$ to every vertex of $\mathrm{K}$. All graphs considered here are simple. Definitions of terms not explicitly given here can be found in [2].

Theorem 2 Let $G$ be connected and $v \in G$. Suppose $\delta \geq 2$. Then $f(v) \geq 1+\delta$. If $f(v)=1+\delta$ then every component of $G-v$ is a $K_{\delta}$.

Proof: To show $f(v) \geq 1+\delta$, first note that it is trivial for $\delta=2$. Suppose the result is true for graphs with $\delta=k$ and let $\mathrm{G}$ have $\delta=k+1$. Let $v$ be a vertex of $\mathrm{G}$ and note that $G-v$ has minimum degree at least $k$. Let $w$ be a neighbor of $v$. Then there is a path of $k+1$ vertices in $G-v$ beginning at $w$. Hence there is a path in $\mathrm{G}$ of $k+1$ vertices in $G-v$ beginning at $w$. Hence there is a path in $\mathrm{G}$ of $k+2$ vertices beginning with $v$ followed by $w$. Now suppose that $f(v)=1+\delta$. By Theorem 1 , there are paths of $2 \delta+1$ vertices in G. Now $v$ must lie in the center of every such path $\mathrm{P}$, or else a path from $v$ to $\mathrm{P}$ followed by the longer subpath of $\mathrm{P}$ would result in a path of more than $1+\delta$ vertices beginning at $v$. Now consider the component or components of $G-v$. Each such component has at least $\delta$ vertices or else the minimum degree of $\mathrm{G}$ would be too small. We now claim that each component $\mathrm{C}$ of $G-v$ has exactly $\delta$ vertices. Let $\mathrm{Q}$ be a longest path in $\mathrm{C} \cup v$ starting at $v$. Certainly the final vertex of $\mathrm{Q}$ has all its neighbors on $\mathrm{Q}$, forcing $\mathrm{Q}$ to have exactly $\delta+1$ vertices. Hence the final vertex of $\mathrm{Q}$ is adjacent to all vertices of $\mathrm{Q}$. Say $\mathrm{Q}: v=v_{1}, v_{2}, \cdots, v_{1+\delta}$. Since $v_{1+\delta}$ is adjacent to every $v_{i}$, it follows that every $v_{i}, i=2, \cdots, \delta+1$, is the final vertex of a longest path in $\mathrm{C} \cup v$ starting at $v$, say $v_{1}, v_{2}, \cdots, v_{i-1}, v_{\delta+1}, v_{\delta}, \cdots, v_{i}$. Hence $v_{i}$ has all its neighbors on $\mathrm{Q}$, so the component $\mathrm{C}$ consists only of the vertices $v_{2}, \cdots, v_{\delta+1}$ and these are all adjacent, proving the theorem.

Theorem 3 If $G$ is connected and $f(G)=2 \delta+1, \delta \geq 2$ then

i) If $G$ has no cut vertex and $n \geq 2 \delta+2$ then $G=H \oplus I$ where $H$ has $\delta$ vertices and $I$ is an independent set.

ii) If $G$ has a cut vertex $v$, then $G$ is the union of copies of $K_{\delta+1}$ with vertex $v$ in common.

\section{Proof:}

i) Since $G$ has no cut vertex, Theorem 4 of [3] assures us that there is a cycle of at least $2 \delta$ vertices. Let $\mathrm{C}$, with vertices $v_{1}, v_{2}, \cdots, v_{2 \delta}$ in cyclic order be such a cycle. First we claim the vertices not on $\mathrm{C}$ are independent, for if $x$ and $y$ are adjacent and neither is on $\mathrm{C}$, then since $\mathrm{G}$ is connected, there is a path from $x$ to a vertex of $\mathrm{C}$. Hence there is a path which includes $x, y$ and all $2 \delta$ vertices of C, contradicting $f(G)=2 \delta+1$. It follows that vertices not on $\mathrm{C}$ have all their 
neighbors on C. Since $n \geq 2 \delta+2$, there are at least two such vertices, say $x$ and $y$. Note that the neighbors of $x$ must be either $v_{1}, v_{3}, \cdots, v_{2 \delta-1}$ or $v_{2}, v_{4}, \cdots, v_{2 \delta}$, for if $x$ were adjacent to two consecutive vertices of $\mathrm{C}$, then $x$ could be inserted between them yielding a cycle of $2 \delta+1$ vertices. In this case, any edge from $y$ to $\mathrm{C}$ would yield a path of length $2 \delta+2$. It follows that every vertex not on $\mathrm{C}$ has either the odd vertices or the even vertices of $\mathrm{C}$ as its neighbors. Suppose that $x$ is adjacent to $v_{1}, v_{3}, \cdots, v_{2 \delta-1}$ and $y$ is adjacent to $v_{2}, v_{4}, \cdots, v_{2 \delta}$. Then $v_{1}, x, v_{3}, v_{2}, y, v_{4}, v_{5}, \cdots, v_{2 \delta}$ is a path of $2 \delta+2$ vertices, contradicting $f(G)=2 \delta+1$. It follows that every vertex not on $\mathrm{C}$ has the same neighbors, say $v_{2}, v_{4}, \cdots, v_{2 \delta}$. We now show that no two odd numbered vertices of $\mathrm{C}$ are adjacent. Suppose $1 \leq i<j \leq 2 \delta-1$ with $i$ and $j$ odd and $v_{i}$ adjacent to $v_{j}$. Then the vertices $x, v_{i-1}, v_{i}, v_{j}, v_{j-1}, v_{j-2}, \cdots, v_{i+1}, y, v_{j+1}, v_{j+2}, \cdots, v_{2 \delta}, v_{1}, v_{2}, \cdots, v_{i-2}$ form a path of $2 \delta+2$ vertices. This again contradicts $f(G)=2 \delta+1$. It follows that the vertices not on $\mathrm{C}$ along with the odd numbered vertices of $\mathrm{C}$ form an independent set $\mathrm{I}$. Let $\mathrm{H}$ be the graph induced by the even numbered vertices of C. All that remains is to show that every odd numbered vertex of $\mathrm{C}$ is adjacent to every even numbered vertex of $\mathrm{C}$. Since an odd numbered vertex of $\mathrm{C}$ can have no neighbor in I, the minimum degree condition forces it to be adjacent to every vertex of $\mathrm{H}$.

ii) Now suppose $f(G)=2 \delta+1$, with $\delta \geq 2$, and suppose G has a cut vertex $v$. Note that every path beginning at $v$ lies (except for $v$ ) entirely in a single component of $G-v$. Since each such component has minimum degree at least $\delta-1$, there are paths of $\delta+1$ vertices beginning with $v$ lying in any component C. If there were a longer path from $\mathrm{v}$ into any such component, then attaching such a path with a path of length greater than or equal to $\delta+1$ vertices from another component of $G-v$ would yield a path longer than $2 \delta+1$ vertices in G. It follows that $f(v)=1+\delta$. By Theorem 2 each component of $G-v$ is $K_{\delta}$. To ensure minimum degree $\delta, v$ must be adjacent to every vertex of every component of $G-v$.

Summarizing, we now know all extremal graphs, G with $f(G)=2 \delta+1$. The connected extremal graphs are those given in Theorem 3 along with the trivial ones with only $2 \delta+1$ vertices.

We noticed that the proof of Theorem 3 is easily modified to provide a characterization of non-hamiltonian graphs which barely miss satisfying Dirac's condition. We are sure this must be known, but we have not found it in the literature. We are grateful to Arthur Hobbs [6], who made us aware of reference [5] where there are results similar to our Theorem 4 . 
Theorem 4 Let $G$ be a graph with $\delta=k$ and $n=2 k+1$ vertices. If $G$ is not hamiltonian then:

i) If $G$ has a cut vertex, then $G$ is the union of two complete graphs $K_{k+1}$ sharing a vertex;

ii) If $G$ has no cut vertex, then $G$ is $H_{k} \oplus I_{k+1}$ where $H_{k}$ is a graph with $k$ vertices and $I_{k+1}$ is an independent set of $k+1$ vertices.

Proof: Note that the degree condition and size of $n$ force $G$ to be connected.

i) This follows immediately from Theorem 1 and Theorem 3 .

ii) In this case, by Theorem 4 of [3], there is a cycle $\mathrm{C}$ of $2 k$ vertices. Let $v$ be the vertex not on C. Say C: $w_{1}, w_{2}, \cdots, w_{2 k}$ in cyclic order. If $v$ were adjacent to two consecutive vertices of $\mathrm{C}, \mathrm{G}$ would have a hamiltonian cycle. Hence we may assume $v$ is adjacent precisely to the $w_{i}$ with $i$ even. As in the proof of Theorem 3, the odd indexed $w_{i}$ are independent. Let $I_{k+1}=v, w_{1}, w_{3}, \cdots, w_{2 k-1}$ and let $H_{k}$ be the subgraph induced by $w_{2}, w_{4}, \cdots, w_{2 k}$. The Theorem follows.

\section{References}

[1] B. Andrasfai, Paths, Circuits, and Loops of Graphs, (Hungarian) Mat. Lapok 13 (1962) 95-107.

[2] G. Chartrand and L. Lesniak, Graphs and Digraphs, 2nd Edition, Wadsworth, Belmont, California, 1986.

[3] G. A. Dirac, Some Theorems on Abstract Graphs, Proc. London Math Soc. 2 (1952) 69-81.

[4] P. Erdös and T. Gallai, On Maximal Paths and Circuits of Graphs, Acta Math. Acad. Sci. Hung. 10 (1959) 337-356.

[5] L. Gordon, Hamiltonian Circuits in Graphs with Many Edges, (unpublished).

[6] A. Hobbs, Private Communication. 